\title{
Alter
}

Revue de phénoménologie

$28 \mid 2020$

La religion

\section{Doubler la métaphysique}

Jean-Luc Marion

\section{(2) OpenEdition}

\section{Journals}

Édition électronique

URL : https://journals.openedition.org/alter/2063

DOI : $10.4000 /$ alter.2063

ISSN : 2558-7927

Éditeur :

Association ALTER, Archives Husserl (CNRS-UMR 8547)

Édition imprimée

Date de publication : 1 novembre 2020

Pagination : 205-226

ISBN : 978-2-9550449-6-4

ISSN : 1249-8947

Référence électronique

Jean-Luc Marion, «Doubler la métaphysique », Alter [En ligne], 28 | 2020, mis en ligne le 22 décembre 2020, consulté le 14 octobre 2021. URL : http://journals.openedition.org/alter/2063 ; DOI : https:// doi.org/10.4000/alter.2063

Ce document a été généré automatiquement le 14 octobre 2021.

Revue Alter 


\title{
Doubler la métaphysique ${ }^{1}$
}

\author{
Jean-Luc Marion
}

\section{Inversion}

Quelle fonction pouvons-nous reconnaitre à la philosophie de la religion? Devons-nous même lui en reconnaître encore une ?

On pourrait en douter, ne serait-ce qu'en considérant son origine, en fait moderne. À proprement parler, il ne saurait y avoir de philosophie de la religion, car elle ne peut intervenir sans la constitution, ou plutôt la reconstitution d'un concept de « religion ». Or ce concept a une origine moderne, rendue possible par l'éclatement de la catholicité occidentale. Certes, depuis les origines, la philosophie s'était enquise du divin, des dieux, puis de Dieu. Mais le concept de "religion» suppose tout autre chose: la comparaison des cultes et des doctrines religieuses, prises dans leur positivité, donc dans leurs divergences, leurs contradictions et leurs apories; ainsi, pour les comprendre, fallait-il supposer un modèle abstrait, susceptible de les comparer, de les unifier ou de les hiérarchiser afin de leur reconnaître ou de leur dénier un statut dans les productions de la culture, sinon du savoir et des sciences. Ainsi comprise, la "religion" pouvait donner lieu à une "philosophie de la religion", puis à des «sciences religieuses». Ce que semble confirmer la première apparition de la Philosophie der Religion sous la plume du jésuite S. von Storchenau, en $1780^{2}$. Mais cette entreprise reste dépendante et contemporaine de la constitution de la metaphysica ellemême, dont elle prolonge l'empire critique dans le domaine que la théologie naturelle avait déjà contesté à la théologie révélée. Cette situation, qui domine encore aujourd'hui, fait de la philosophie de la religion (comme de l'histoire des religions ou même, pour une bonne part, des "sciences religieuses") une extension du projet philosophique, donc en fait métaphysique, dans un champ périphérique, comparable à d'autres (philosophie des sciences, philosophie politique, philosophie de l'art, etc.). La philosophie de la religion consiste alors en une philosophie appliquée, entre autres objets, à « la religion », qu'elle tente de ramener à la raison. 
Ce modèle, que Hegel a positivement accompli (et que Marx et Nietzsche ont simplement retourné), se trouve pourtant aujourd'hui en crise. Non pas d'abord en conséquence d'une improbable et improuvable crise du divin, des dieux ou de Dieu, comme nous nous le faisons accroire sans bien comprendre ce que nous disons, mais suite à l'indétermination du concept même de "religion " (et encore plus de «la religion ») qu'on présuppose en toute philosophie de la religion. Il y a plus : au fondement de l'indétermination du concept de " religion » se repère l'indétermination, autrement inquiète, de la philosophie qui prétend déployer ce concept; plus exactement l'indétermination de la détermination métaphysique décidée par et pour la philosophie. L'imprécision des meilleures tentatives récentes, menées pourtant par les meilleurs esprits, pour ne fût-ce qu'esquisser les principes et contours d'une philosophie de la religion résulte essentiellement de l'impuissance $\mathrm{du}$ projet métaphysique qui les sous-tend, ou de son absence totale. Quelle raison philosophique (ou métaphysique) peut-on reconnaître comme qualifiée pour rendre raison d'une réalité aussi indéterminée pour elle et en soi que « la religion »? Quelle logique, quels principes et quels a priori peuvent légitimement prétendre ramener à l'intelligibilité un champ - le divin, les dieux ou Dieu - à propos duquel notre théorie avoue tout ignorer et même souvent devoir tout ignorer ? La crise de la philosophie de la religion apparaît soudain comme l'indice et le symptôme de la crise de la raison philosophique ellemême, autrement dit de sa détermination métaphysique. Schelling, lorsqu'il tentait de libérer la philosophie de la metaphysica (dans son langage de renverser la philosophie négative en philosophie positive), n'hésitait pas à inverser les termes de la question :

Mais aussi, en régime de christianisme, on ne doit pas se demander comment doisje penser, pour le mettre en accord avec une philosophie quelconque? Mais inversement, quel doit être le type d'une philosophie, pour qu'elle puisse aussi prendre sur elle le christianisme et le comprendre par concept (an sich aufnehmen und begreifen) $)^{3}$ ?

Il se pourrait désormais que le succès de toute tentative sérieuse d'esquisser une philosophie non-métaphysique se mesure à sa capacité à parler correctement et dignement du sacré, des dieux et de Dieu. Cette question n'offre plus aujourd'hui une région périphérique à la rationalité, éventuellement facultative, comme une colonie lointaine, voire une terra incognita où exporter, non sans violence parfois, les certitudes du rationalisme bien établi en métropole. Cette question devient la pointe avancée de la rationalité, où celle-ci éprouve ses forces, ses faiblesses et toujours ses limites en les soumettant à l'épreuve dans les conditions les plus extrêmes, les conditions de l'inconditionné. Inconditionné en effet, et d'un type nouveau, puisqu'il fait exception à ce que nous conditionnons, en s'imposant comme ce que nous ne conditionnons plus. Cet $x$ non conditionné par nous, nous en faisons l'expérience par les conditions qu'il nous impose d'ailleurs, quelque nom que nous donnions à cet ailleurs. Ainsi la posture de la philosophie de la religion s'inverse-t-elle : elle n'assure plus, au nom de la metaphysica, le tribunal de la critique rationaliste sur le territoire dissident, régional et supposé ontique de " la religion »; elle désigne, en termes encore imprécis, la mise en crise de la rationalité et de sa prétention à tracer des limites selon ses concepts a priori.

5 Termes encore imprécis, en effet. Car cette fonction critique ne peut pas s'exercer par une instance aussi mal définie que " la religion » (encore moins entendue au pluriel, à partir d'un commun dénominateur adéquat à aucune d'elles) ; d'autant que toute définition possible de « la religion » met précisément en œuvre des a priori, ou du moins des décisions qui en construisent sans elle le concept. Cette fonction critique ne peut 
provenir par hypothèse que d'ailleurs. Toute la difficulté ne consiste qu'à déterminer comment, d'où cet ailleurs peut advenir. Il devrait, en tout cas, s'agir d'un fait de la raison, au sens où la loi morale s'impose de facto à la raison pratique, de l'exérieur, sans autre condition de possibilité que sa propre possibilité, aussi impossible qu'elle nous apparaisse - au point qu'elle nous "humilie ». Mais, comme il n'en va pas ici de la raison pratique, mais de ce qui se dissimule encore sous le terme inapporprié de «la religion ", il faut chercher au-delà le fait, dont la raison non-métaphysique, se trouve en manque, sinon déjà en quête. Ce terme, ou plutôt ce point de visée la théologie, en particulier la théologie chrétienne pour autant qu'elle garde encore aujourd'hui ses ressources originairement non-métaphysiques, peut le préciser: il s'agit de la Révélation, comme le fait a priori provenant d'un ailleurs radical. La philosophie de la religion ne trouve ou retrouvera aujourd'hui un rôle décisif que dans la mesure stricte où elle se concentrera sur l'affrontement entre la metaphysica et la Révélation.

\section{Situation}

Le rapport entre la théologie judéo-chrétienne et ce que l'on nomme la métaphysique a toujours été problématique. Il devient aujourd'hui conflictuel et crucial. C'est pourquoi il suscite la polémique, toujours recommencée et toujours manquée. Il s'en trouve deux motifs principaux.

7 L'un se repère avec évidence : aucune des deux figures extrêmes qui pourraient définir leur rapport ne convient. On ne peut soutenir que le christianisme puisse et doive aujourd'hui plus que dans le passé s'identifier absolument à une doctrine de l'être, ne fût-ce que parce que, d'une manière ou d'une autre, si Dieu est, il n'est donc pas au même sens où sont les choses; ou parce qu'il ne va pas de soi que la métaphysique reste encore aujourd'hui en état de penser la question de l'être. On ne peut pas plus soutenir que le christianisme ne doive concevoir la radicale altérité de Dieu, qu'en renonçant à la penser par concept, ou du moins à la penser au niveau de la raison. Ces deux extrêmes, rationalisme univoque ou manichéisme conceptuel, entérineraient une égale défaite de la rationalité et de la foi.

Mais un autre motif, moins visible peut-être, mais plus contraignant, s'ajoute au premier. Car pouvons-nous aujourd'hui admettre, comme une évidence, que la metaphysica ait une signification si décidée et offre de tels principes de rationalité qu'elle puisse fonder le discours de la théologie? Nul d'entre nous, sans doute, ne pourrait souscrire sans réserve à la déclaration que Suarez inscrivait en prologue de ses Disputations métaphysiques. Elle mérite qu'on la relise :

Bien que la théologie divine et surnaturelle s'appuie sur des principes révélés par Dieu dans la lumière divine, elle s'aide aussi des vérités connues par la lumière de la nature et s'en sert comme ses serviteurs et quasi ses instruments pour améliorer ses raisonnements et éclairer les vérités divines. [...] Car, puisque, quand on discute des mystères divins, interviennent ces dogmes métaphysiques (haec metaphysica dogmata) sans la connaissance et l'intelligence desquels on ne peut qu'à peine, ou même pas du tout (vix, vel ne vix quidem) traiter ces très hauts mystères selon leur dignité, j'ai été contraint de souvent [...] mêler aux choses divines et surnaturelles des questions inférieures. [...] Car ces principes et vérités métaphysiques forment un tel tout cohérent avec les conclusions et les raisonnements théologiques (principia et veritates metaphysicae cum theologis conclusionibus ac discursibus ita cohaerent), que, si on ôtait la science et la parfaite connaissance des premières, il 
serait aussi nécessaire que la science des secondes s'effondre assurément (nimirum) ${ }^{4}$. base solide, voire des principes fondateurs à la rationalité théologique, la metaphysica offrirait plutôt une hypothèque et aporie, qui viennent s'ajouter à celle que constitue son rapport complexe et discuté au christianisme.

semblent convenir, ouvre un théâtre pour toutes les discussions et même toutes les disputes. Ce qui indique, par contraste, la nécessité de redéfinir les termes de la question. Et, pour cela, commençons par des distinctions pour ainsi dire formelles, et, comme telles, admissibles par tous, sinon indiscutables.

\section{Distinctions}

11 D'un point de vue historique d'abord, il faut remarquer que la metaphysica ne recouvre pas toute la philosophie. Personne ou presque avant Thomas d'Aquin, qui lui-même ne formalise pas la metaphysica comme telle, n'a tenté d'articuler cette dernière à la philosophie en général. C'est à Jean Duns Scot qu'on doit l'émergence de la metaphysica au rang de scientia transcendantalis ${ }^{5}$; c'est surtout à Suarez que l'on doit sa domination comme "système de la métaphysique », tel qu'il s'est développé jusqu'à Baumgarten et Kant, et même, en un autre sens, Fichte et Hegel. Mais dès Schelling, qui la met en cause sous le titre de philosophie négative, elle entre dans une période de conflit, d'autant plus réaffirmée qu'elle se redéfinit sans cesse et reste sous pression des critiques toujours plus puissantes de Kierkegaard, Marx et Nietzsche. La possibilité d'une philosophie non-métaphysique traverse donc tout le XIX jusqu'à la phénoménologie et au Cercle de Vienne. La tentative récente de répertorier une pluralité de «métaphysiques rebelles» confirme $a$ contrario cette unicité historique de fait ${ }^{6}$. Car, entre les trois modèles à l'origine concurrents au Moyen Âge - la science de Dieu, l'articulation de l'ontologie à la théologie, la science transcendantale (soit pour simplifier Bonaventure, Thomas d'Aquin et Jean Scot), seule la dernière, en tant que la scientia transcendentalis, a utilisé et imposé le terme de metaphysica. Et c'est dans son seul sillage, repris dans l'école cartésienne, mais surtout dans la scolastique calviniste du $\mathrm{XVII}^{\mathrm{e}}$ siècle, que naquit le néologisme tardif d'ontologia, ignoré des médiévaux comme d'Aristote et de ses commentateurs. La métaphysique, à savoir en réalité la metaphysica, ne résulte donc que d'une généalogie hésitante et récente.

Cette linéarité fragile se redouble d'une étroitesse d'emploi. En effet, durant l'époque moderne, celle même où domine la metaphysica, nombre de philosophes, et non des moindres, hésitent à s'en revendiquer, même si, d'une manière ou d'une autre, des interprétations modernes peuvent, à la suite de Heidegger, les inclure sous la figure d'une constitution onto-théo-logique : ainsi Descartes, Spinoza, Locke, Hume et même Leibniz. À partir de Fichte, plus encore que Kant, le terme se trouve déjà sous suspicion et tend à le céder à d'autres titres - transzendentale Philosophie, Wissenschaftslehre, System, Erkenntnistheorie, etc. Quant à l'époque contemporaine, l'hésitation et l'indécision, voire les volte-face n'y manquent pas: Husserl a attendu les Méditations Cartésiennes pour admettre le terme, d'ailleurs avec réticence et prudence. Heidegger, qui l'avait franchement assumé dans Kant und das Problem der Metaphysik, puis dans l'Einführung in die Metaphysik et jusque dans «Was ist Metaphysik?», finit par s'y opposer et même 
« la laisser à elle-même ». Lévinas n'a pas tranché nettement, s'opposant à la totalité, mais la sauvegardant par l'infini. Derrida l'a destitué très explicitement, mais certains (dont je suis) ne sont pas sûrs qu'il en soit resté in fine indemne. Exemplaire apparaît enfin le retournement de la position initiale de la philosophie analytique : alors que Carnap récusait Heidegger compris comme le métaphysicien par excellence (Die Überwindung der Metaphysik durch die logische Analyse der Sprache), ses successeurs contemporains (Armstrong, etc.) restituent le programme métaphysique, tout en le réduisant à une ontologie de l'objet et en atténuant les exigences fondationnelles de ses principes. La "métaphysique " ne bénéficie, aujourd'hui, que d'une instauration aussi fragile et mouvante que celle de la metaphysica.

Une fois constatées, au simple point de vue de l'historien, cette étroitesse généalogique et cette fragilité d'instauration doivent à la fin s'expliquer par des motifs conceptuels. Plusieurs sont apparus avec une évidence qui n'appelle, ici du moins, aucun commentaire développé. - (a) La metaphysica ne considère pas l'être, mais seulement l'être de l'étant, voire en fait l'étant seul et en tant que tel, ens ut tale; or, pour mériter le titre de science de l'être (comme le voudraient ses partisans contemporains), il eût fallu qu'elle considérât non pas l'ens in quantum ens, mais précisément l'ens in quantum esse (tel fut le diagnostic de Heidegger, mais aussi, à sa manière, de Gilson). Et précisément elle a ignoré ou manqué la question de l'esse. - (b) Plus, en considérant l'étant seul, la metaphysica ne l'a pas considéré uniquement ni longtemps, ni d'abord comme un étant (ens in quantum ens), mais comme un étant connaissable et représentable (ens ut cognitum, ut intelligibile, ut cogitabile), selon la décision canonique des instaurateurs de l'ontologia:

Cette Métaphysique comme on l'appelle habituellement, mais qu'il faudrait plus exactement appeler Ontologie ou science catholique, est nommée une science universelle et philosophie universelle [...] elle dénote tout ce qui peut se cogiter (l'intelligible, disent certains par souci de distinction). [...] omettant donc quelques traits de l'étant pris dans les deux premières acceptions [quelque chose \& substance/ accident], nous commencerons la philosophie universelle par l'étant cogitable, tout de même que, quand elle commence par le singulier, la philosophie première ne considère rien avant l'esprit cogitant ${ }^{7}$.

On notera aussitôt que cette réduction de l'étant à l'étant comme cogitabile rend presque banale et évidente la déclaration un peu trop solennelle de Kant : «[...] le nom orgueilleux d'ontologie, qui prétend donner des connaissances synthétiques a priori des choses en général dans une doctrine systématique (par exemple les principes de la causalité), doit céder la place à celui plus modeste de simple analytique de l'entendement pur $»^{8}$. En fait, l'ontologia avait d'emblée, sans attendre sa critique par Kant, réduit l'ens à ce que l'entendement pur pouvait a priori en concevoir (en l'occurrence sa possibilité, fixée par les conditions a priori de l'expérience). Et il n'y a rien non plus d'étonnant à ce que l'ontologia de la métaphysique aboutisse ainsi, toujours et encore aujourd'hui, à une ontologie de l'objet, puisque les conditions de l'expérience sont aussi et du même coup les conditions des objets de l'expérience. Le succès de la métaphysique ne fut réel qu'en se restreignant à la connaissance des objets, qu'elle constituait précisément afin de les connaître. - (c) Le privilège accordé à l'étant sur l'être en lui peut enfin lui conférer une fonction privilégiée à l'égard de tous les étants : il lui revient, au lieu et à la place de l'être, lui-même réduit au silence, de déployer tous les autres étants; il l'a de fait accompli sous le mode (lui aussi strictement ontique et épistémique) de l'explication et de la fondation; l'étant vire alors à l'étant suprême (ens supremum), qui a pour fonction de fonder la totalité des 
étants (à titre de principium, de causa, donc en s'érigeant lui-même finalement comme causa sui). Ainsi l'onto-théologie permet, entre autres conséquences, une détermination idolâtrique de Dieu (d'où les dénonciations de Nietzsche et déjà de Pascal). En conséquence, ces faillites paraissent si massives, que presque tous les partisans contemporains d'un retour à une métaphysique entendent bien ne pas en rester les otages. Ou bien, si métaphysique il doit encore y avoir (par exemple une "métaphysique de l'Exode »), cela ne se pourra qu'en rupture avec la metaphysica, telle qu'elle s'est trouvée historiquement réalisée ; car, comme Gilson le premier l'a constaté, "Tout se passe comme si l'histoire de la méta-physique était celle d'une science qui se trompe continuellement d'objet $»^{9}$.

S'il y a donc et au dire de ses partisans eux-mêmes une crise de la métaphysique, quelles ressources demeurent pour lui substituer une autre métaphysique? Une autre métaphysique - qu'on n'entendra non point comme autre que la métaphysique, mais comme une metaphysica autrement pensée, simplement rectifiée et mieux recommencée. Ces rectifications furent en effet toutes tentées et par les meilleurs philosophes. On peut en distinguer au moins trois types. - (a) Les rectifications internes à l'horizon métaphysique, qui conduisent à considérer l'ens selon un autre transcendantal que celui de l'être, par exemple l'un (ainsi S. Breton), le bien (ainsi Lévinas et plus récemment Brague), ou le beau (ainsi H. U. von Balthasar). - (b) Les rectifications régressives, qui persistent à demeurer dans l'horizon ininterrogé de l'objectité et rétablissent décidément une ontologie de l'objet; ce qui suppose non seulement le maintien et l'extension de la logique, mais de n'en jamais interroger l'essence ni la fonction ${ }^{10}$. - (c) Ou, dernière posture, la sigétique: l'exigence métaphysique ne s'atteste plus que dans le silence fait sur son territoire et son projet; il peut s'agir d'un silence explicite (Wittgenstein), ou d'un silence lui-même pour ainsi dire réduit au silence par le bruit de la déconstruction (Derrida). - Il reste enfin une dernière rectification, mais qui englobe toutes les précédentes : réduire l'entreprise de la métaphysique à sa dimension transgressive de scientia transphysica, telle que prétend l'ouvrir la «fonction méta- » (Breton, Ricoeur, Greisch, etc.). Une telle « fonction méta- » indiquerait dès lors le caractère propre de la métaphysique: en contraste avec l'horizon déterminé de toute science particulière, la métaphysique se signalerait par sa puissance de trans-gression, de dépassement de l'horizon déterminé de chaque science vers un horizon sans détermination, autrement dit (puisque par définition tout horizon délimite ou se détermine par une limite) vers un non-horizon ; la métaphysique devrait se penser comme une science de l'étant en tant que $x$, ni étant, ni rien, ni être, ni autrement qu'être, science de l'en-tant-que comme tel pour et par une herméneutique sans fin. L'illimitation de la fonction méta-implique donc l'indétermination de son nonhorizon. Mais alors une objection se dessine, précisément en vertu de cette réinterprétation de la metaphysica : $s i$ « métaphysique » finit par signifier dans tous les cas une méta-métaphysique ${ }^{11}$, et si donc la restauration de la métaphysique passe par son dépassement continu et son effacement toujours repris, pourquoi maintenir le nom qu'on lui demande précisément de rayer et transgresser - celui de la metaphysica, la seule historiquement achevée? Pourquoi garder le nom, s'il faut de toute façon dépasser la chose? Que gagne-t-on à nommer encore métaphysique ce dont on convient qu'il faut se défaire ${ }^{12}$ ?

D'où cette première conclusion : il faut en général, et donc en particulier pour la pensée chrétienne, doubler la métaphysique, la doubler comme un marin double un cap - pour le 
défier, pour s'en libérer, pour ouvrir l'horizon sur un autre océan sans limites apparentes. Dépasser la métaphysique et la question de l'être de l'étant voudrait ainsi dire passer outre la métaphysique (et son privilège conféré à l'étant, à l'objet et au pensable), voire outrepasser la Seinsfrage (et le privilège qu'elle concède à la persistance de la présence) comme on passe, contourne et dépasse le Cap Horn. D'ailleurs, comment ne pas admettre qu'en fait aucun de nous ne pratique plus la métaphysique, au seul sens historique précis que nous lui connaissions, celui de la metaphysica. Sous le nom de "métaphysique", même et surtout ses partisans les plus résolus visent depuis longtemps tout autre chose que ce qu'en fut et en reste l'accomplissement historique.

\section{Limites}

17 L'entreprise de la metaphysica implique par définition des limites, etc, a priori. Pour doubler la métaphysique, ne serait-ce que pour en redoubler le meta-, il faut donc déplacer ou franchir des limites. Et pour les franchir, il faut d'abord les identifier. Or, on voit clairement que l'extension du front et du champ de l'expérience s'arrête, en stricte métaphysique, aux bornes du possible et de l'impossible et à leur distinction originaire. Un méta-, un pas hors du possible tel que la métaphysique l'entend et le colonise, devrait (à s'en tenir à l'indice très tôt fourni par la métaphysique elle-même lors de son institution moderne) affronter la polémique sur le miracle, polémique dont la violence tenait précisément à la mise en jeu du possible et de l'impossible comme frontière inavouée mais pourtant inévitable de l'a priori. Hume définit le miracle comme ce qui, très précisément, ne peut se penser métaphysiquement puisqu'il ne consiste qu'en «[...] a violation of the laws of nature $»^{13}$. Cette exclusion bénéficiait, à l'époque, de l'apparente évidence de ses assomptions: il y a des lois de la nature, certes pas encore toutes établies, ni ne gouvernant pas la totalité des phénomènes, mais pourtant de droit censées y parvenir, à terme. Et ceci, d'autant qu'on pouvait aussi les assimiler aux supposées "lois de l'ordre", dans lesquelles Malebranche reconnaissait le Verbe divin lui-même. Mais l'histoire des sciences a depuis bien établi qu'à proprement parler il ne se trouve pas de "lois de la nature", sinon à titre d'hypothèses toujours révisables. Et ceci, d'autant plus qu'il ne se trouve pas de "nature", sinon ce que l'arraisonnement technologique défait et refait à sa mesure. En conséquence, pour le meilleur et pour le pire, la vision contemporaine ne peut et ne prétend plus se fixer de limites du possible. La critique du miracle appartient donc à l'histoire de la metaphysica, et disparait dans l'horizon contemporain du nihilisme. La philosophie, ne pouvant aujourd'hui plus supposer acquise la différence entre le possible et l'impossible, doit l'affronter comme une question. Une question pour la philosophie certes, mais pas seulement pour elle.

Or, il se trouve que cette question - doubler les limites de la possibilité - concerne aussi la pensée du christianisme. Ou du moins devrait la concerner au premier chef, si le christianisme pensait vraiment ce qui lui revient d'incarner, au lieu, comme le constatait Péguy, de s'y dérober: "Quand aurons-nous le désétablissement de la métaphysique ? $\aleph^{14}$. C'est ce désétablissement que sans doute visait aussi $\mathrm{S}$. Weil sous le titre de "nettoyage philosophique de la religion catholique $»^{15}$. Mais, si la théologie chrétienne (et juive) se résolvait à doubler le cap de la metaphysica, encore faudrait-il déterminer la limite en question, et qui aurait-il qualité pour la doubler. 
La réponse à cette question pourrait s'esquisser à partir de deux constatations, l'une philosophique, l'autre théologique, et de leur rapprochement. - (a) En termes philosophiques, on a constaté que la décision initiale de la metaphysica consiste à définir l'ens par la possibilité. Ou bien négativement - «Ce qui est impossible ne peut exister » - ou bien positivement - «Ce qui est possible peut seul exister $»^{16}$ - étant entendu que la possibilité elle-même se définit par la représentation pour nous non-contradictoire : «Le non-rien est quelque chose. Le représentable, à savoir ce qui n'englobe aucune contradiction, ce qui, quel qu'il soit, n'est pas A et non-A, cela est possible $»^{17}$. Kant confirme et redouble ce principe en reconduisant le couple possible/impossible au concept suprême d'objet : " Le concept le plus élevé, par lequel on a coutume de commencer une philosophie transcendantale, est généralement la division entre le possible et l'impossible. Mais, comme toute division suppose un concept divisé, on doit en admettre encore un autre, plus élevé, et c'est le concept d'un objet en général » ${ }^{18}$. En fait, Kant manifeste clairement que la possibilité, conçue comme condition de toute représentation pour un esprit fini dans les limites a priori de l'expérience, ne peut concerner justement que des objets. Transgresser la limite entre les concepts de possible et d'impossible implique donc de contester aussi l'irréductible priorité du concept d'objet. Philosophiquement, la limite à franchir comme une frontière coïncide avec la ligne qui sépare le possible et l'impossible.

Or (b) il se trouve, cette fois-ci en termes bibliques, donc théologiques, que le propre de Dieu consiste aussi et également (ce qui ne veut pas dire univoquement) à ne pas dépendre de la distinction ni de l'opposition entre le possible et l'impossible : «à Dieu rien d'impossible $»^{19}$, et ceci contrairement à l'homme, pour qui justement le possible se heurte toujours, en dernière instance, à l'impossible (ne fût-ce que sa mort) : pour le mortel, le possible se définit toujours en droit comme le non-encore- impossible, qui nécessairement finira pas le devenir - impossible. Tel que l'annoncent les prophètes et que le révèle Jésus-Christ, Dieu se caractérise au contraire par la levée de la limite de la metaphysica: pour lui, possible et impossible ne se distinguent pas (même si les extrêmes de l'[im-]possible ne coïncident pas, pour lui, avec ce qu'ils paraissent chez nous). C'est cette coïncidence qu'avait admirablement vue et soulignée l'un des rares penseurs contemporains de l'établissement de la metaphysica à n'y avoir pas souscrit, Nicolas de Cues :

D'où il suit que, puisque rien n'est impossible à Dieu, il faut que ce soit à travers les choses qui sont dans ce monde impossibles que nous dirigions notre regard vers celui chez qui l'impossibilité est une nécessité - tout de même que l'infinité en acte est impossible dans ce monde, ainsi la grandeur sans fin est la nécessité même qui rend nécessaire que le non-étant ou rien soit ${ }^{20}$.

Ou encore :

C'est pourquoi je te rends grâce, mon Dieu, de m'avoir dévoilé qu'il n'y a pas d'autre voie pour accéder à toi, que celle qui paraît radicalement inaccessible et impossible à tous les hommes, mêmes aux plus savants des philosophes, puisque tu m'as montré que l'on ne peut te voir ailleurs que là où l'impossibilité advient et se met en travers, ubi impossibilitas occurrit et obviat ${ }^{21}$.

22 Ainsi l'espace où pourrait, peut-être, prendre un sens la question du Dieu tel qu'il se révèle en Jésus-Christ ne s'ouvre-t-il qu'une fois le possible et l'impossible outrepassés (donc une fois l'objectité elle aussi outrepassée), bref une fois outrepassé notre domaine, celui que nous suffisons à régir (du moins pouvons-nous le revendiquer). L'espace où Dieu se manifeste s'ouvre, pour nous, là où l'impossible devient, ou plutôt peut devenir 
possible, c'est-à-dire où nous, nous ne pouvons pas faire un pas, car ce « sol est une terre sainte » (Exode 3, 5).

Nous en sommes sans doute arrivés au point où il devient raisonnable de travailler (de se laisser travailler par) l'impossible. Donc d'écouter sérieusement l'avertissement de Heidegger : «Das Unmögliche ist des Menschen höchste Möglichkeit, l'impossible est la plus haute possibilité de l'homme »²2. (G.1. 96, p. 273). Et le mot d'ordre de Mallarmé : « Il n'y a de réalisable que l'impossible $! »^{23}$.

\section{Les ordres}

Mais il n'est pas impossible d'atteindre l'impossible. Car la meta-physica le définit très aisément par opposition à ce qu'elle pense comme le possible - à la possibilité d'une essence, selon le critère de la contradiction logique de A envers non-A ; en corrélat, la définition de l'impossibilité d'une existence par l'impuissance de l'effectivité (il n'est pas possible que A devienne effectif) se borne à décliner cette contradiction depuis l'essence jusqu'à l'existence; ou plus précisément, elle constate l'insuffisance de la cause à produire l'effet, donc finalement entérine l'inadéquation de la raison suffisante à une telle existence. Ces lois logiques, qui précisément définissent la pensée finie, la théologie chrétienne ne prétend certes pas les modifier, ni s'en dispenser : elle envisage plutôt de les contourner et d'en doubler le cap en surdéterminant cette logique même. Non pas vers une absence de logique (situation de droit inconcevable), mais vers une redéfinition de ce que le logos implique et déploie en fait de logique. Car la théologie chrétienne se caractérise, entre autres singularités, par la dénomination de Dieu comme Logos; cette dénomination permet d'envisager de redoubler un logos par un autre, éventuellement de redoubler une logique par une autre - en sorte que certaines impossibilités se renversent en possibilités, selon une autre logique que celle de la formalité métaphysique. Comment thématiser ce franchissement et ce renversement? Comment identifier la limite où, comme sur une ligne de partage des eaux, le possible et l'impossible se divisent? En considérant sérieusement la formule qui désigne le fonds de la révélation biblique : "Dieu est agapê » (1 Jean 4, 8 \& 16) et formalise le fait que Jésus Christ « ...ayant aimé (agapêsas) les siens qui étaient dans le monde, les aima jusqu'au terme (eis telos hêgapêsên autous) » (Jean 13, 1). La question dès lors se précise : pour atteindre le point à partir duquel on pourrait doubler le cap de l'impossible (donc aussi de l'objectité), il faut redoubler la logique de la metaphysica par une logique de l'agapê.

On doit à Pascal, confronté à la fois à la fondation cartésienne de la cogitatio dans l'ego et (par le biais des controverses sur la grâce) aux premières conséquences de l'instauration du système de la metaphysica, d'avoir génialement et sans doute définitivement marqué cet écart et fixé l'unique moyen de le franchir. La doctrine des trois ordres montre en effet que l'agapê, ici nommée la charité, constitue à la fois le fonds propre de la pensée chrétienne et l'unique moyen d'y accéder. Relisons le texte qui redouble la distinction entre l'étendue et la cogitatio cartésienne (et donc l'ens ut cogitabile sous la garde du possible) par l'écart incommensurable entre cette même cogitatio et la charité :

La distance infinie des corps aux esprits figure la distance infiniment plus infinie des esprits à la charité, car elle est surnaturelle. / Tout l'éclat des grandeurs n'a point de lustre pour les gens qui sont dans les recherches de l'esprit. / La grandeur 
des gens d'esprit est invisible aux rois, aux riches, aux capitaine, à tous ces grands de chair. / La grandeur de la sagesse, qui n'est nulle sinon de Dieu, est invisible aux charnels et aux gens d'esprit. Ce sont trois ordres différents, de genre. [...] Tous les corps, le firmament, les étoiles, la terre et ses royaumes, ne valent pas le moindre des esprits. Car il connait tout cela, et soi, et les corps rien. / Tous les corps ensemble et tous les esprits ensemble et toutes leurs productions ne valent pas le moindre mouvement de charité. Cela est d'un ordre infiniment plus élevé. / De tous les corps ensemble, on ne saurait en faire réussir une petite pensée. Cela est impossible et d'un autre ordre. De tous les corps et esprits on n'en saurait tirer un mouvement de vraie charité, cela est impossible et d'un autre ordre surnaturel ${ }^{24}$. déploie qu'en esquissant une doctrine, autonome et forte, de la charité (ne disons pas en la construisant, car la pensée chrétienne se trouve plutôt elle-même construite par cette instance). D'où l'exigence impérative de ne pas abandonner le phénomène érotique à la metaphysica (pas plus qu'à la psychanalyse, à la " fable mystique », encore moins aux supposées spiritualités), mais le penser à fond et donc à partir de l'agapê, telle que performée « jusqu'au terme » (Jean 13,1) par le Christ. La tâche et la condition de possibilité de la pensée chrétienne consistent, avant toute chose et 
inconditionnellement, à accéder à une pensée conceptuelle, donc trinitaire de l'agapê. Mais, il faut l'admettre une bonne fois, cette question ne se confond en rien avec celle de la métaphysique, ou plutôt la confond en la rendant confuse : être ou ne pas être, telle n'est pas la question qui suscite, identifie et justifie la pensée chrétienne; dès qu'au contraire elle reste sur ce fonds métaphysique, elle ne pense plus chrétiennement et même ne pense plus du tout ; elle mime, récupère et répète ce qui lui reste étranger. Il ne s'agit jamais, pour la pensée chrétienne, de penser métaphysiquement Dieu, ni d'élaborer une « métaphysique de la charité »- formules, certes courantes, mais, à bien y songer, en droit contradictoires, ou simples non-sens. Doubler la métaphysique ne demande donc ni de l'oublier, ni de la critiquer, mais de redoubler cette science "toujours recherchée et toujours manquée ${ }^{26}$ sur l'étant en tant qu'étant par une pensée de la charité accomplie en tant que telle (Jean 19, 28). Entre ces deux entreprises, il ne se trouve ni concurrence, ni alliance, ni conflit, ni compromis - car il s'agit de deux ordres infiniment différents. Et une même pensée ne doit pas s'aventurer à servir deux ordres ensemble.

\section{Doubler la métaphysique : la faire servir à un autre dessein que le sien}

Mais qu'en est-il du besoin de la raison, qui persiste ? En effet, non seulement, il se pourrait que la raison ne puisse se dispenser de chercher (et trouver) des fondements à ses objets, en sorte qu' " [...] on reviendra toujours à la métaphysique comme à la bienaimée, avec laquelle on s'était brouillé $»^{27}$. Mais alors, même si la raison parvenait à se libérer de sa nostalgie et de ses réflexes métaphysiques, la philosophie d'après la metaphysica n'en demeurerait pas moins une exigence légitime de la pensée. Nous avons déjà identifié ces deux entreprises, en elles-mêmes parfaitement admissibles : ou bien une (néo-)métaphysique, qui repose sur et aboutit à une ontologie de l'objet (le plus souvent), ou bien une ontologie de l'étant, qui l'envisagerait sous un autre nom, voire sous une forme révisée. Cependant ces deux entreprises, à supposer qu'elles aboutissent, ne concerneraient pourtant encore en rien la pensée chrétienne, qui se déploie, elle, à partir de ce que Schelling appela un «imprépensable» (ein Unvordenkliches), qui lui vient d'ailleurs, comme un a posteriori irréductible parce que donné, l'agapê comme son fonds propre. En ce sens, elle prend statut de théologie, directement concentrée sur le troisième ordre, sans aucune norme métaphysique, mais suivant une norme trinitaire puisque christologique. Il y aurait - il y a donc - deux ordres, qui autorisent et réclament deux modes de pensée et deux logiques irréductibles : soit l'« esprit » (ensemble la metaphysica, la métaphysique, la philosophie éventuellement post-métaphysique), soit la "charité » (la logique du phénomène érotique, la théologie christologique et trinitaire), qui double la métaphysique en redoublant les deux premiers ordres par le troisième.

Pourtant, cette césure n'interdit pas un effet en retour de la " charité » sur l'« esprit », qui articule entre eux ces deux ordres. Car Pascal maintient explicitement que, si chaque ordre supérieur reste invisible à l'ordre inférieur (les deuxièmes et troisièmes invisibles au premier, le troisième aux deux premiers), chaque ordre supérieur voit et " juge » celui ou ceux qu'il domine. Cette visibilité, non-réciproque mais unilatérale, qui ne fonctionne que de haut en bas et jamais de bas en haut, permet en effet de renverser le point de vue entre les ordres, et de voir, donc de décrire chaque ordre inférieur du 
point de vue de tout ordre supérieur. Ainsi les «corps " peuvent-ils se décrire et juger du point de vue des "esprits " et de la "charité ", comme aussi bien les " esprits " (ainsi la metaphysica, la philosophie, la Seinsfrage, etc.) du point de vue de la "charité ». Il devient donc pensable de comprendre et d'interpréter des concepts de l'« esprit ») à partir de ceux de la "charité", autrement dit de les soumettre à une réduction érotique, pour, au fil conducteur de l'herméneutique qui en résulte, les revoir (et voir ce qu'ils pourraient en dernière instance permettre d'y concevoir) ${ }^{28}$ dans la lumière de l'agapê. Il ne s'agit pas de réinstaurer in fine la moindre continuité entre deux ordres infiniment incommensurables, mais, à l'inverse, d'en constater l'écart en découvrant comment le troisième ordre ne voit pas le deuxième comme celui-ci se voit et y voit autre chose que ce qu'il montre. L'herméneutique que déploie le regard de la «charité » sur l'«esprit » n'y voit pas ce que l'« esprit» voit de lui-même, mais y discerne un symptôme de la charité, un état de charité qui s'ignore. Au lieu de convoquer les facultés au tribunal de la raison, la philosophie se trouve ainsi elle-même convoquée au tribunal de l'agapê. Non qu'il s'agisse d'" introduire la lutte des classes dans la théorie » (ce qui reviendrait à juger le deuxième ordre par le premier, le supérieur par l'inférieur, la superstructure par l'infrastructure, démarche caractéristique de l'idéologie, antithèse du modèle pascalien), mais d'introduire la lux redarguens $\mathrm{du}$ troisième ordre dans le champ du deuxième ordre, qui n'admet que la veritas lucens ${ }^{29}$.

Ainsi, par un exemple privilégié, la Seinsfrage (et même la métaphysique) peut paraître sous une lumière non pas ontologique, mais - sit venia verbo - érotique. Il suffit de s'interroger sur l'équivalence entre ousia et parousia, qui présuppose qu'être implique de persister dans la présence, donc qu'être implique la possession de la présence comme un fonds à s'approprier; dès lors la persistance du fonds approprié (parousia, Beharrung) vire à la persévérance (conatus in suo esse perseverandi), donc à la volonté de puissance comme volonté exclusive de soi et de son accumulation indéfinie. Le nihilisme (ontique) apparaît alors comme un effet et un symptôme du déni de l'agapê, elle-même alors réduite dans le monde à sa figure kénotique ${ }^{30}$.

Ainsi encore la question de Dieu se modifie radicalement - et il y va d'un décentrement ou plutôt d'un recentrement complet des objectifs de la philosophie de la religion en son acception traditionnelle. Parce que la question de l'être n'a en effet que peu, ou pas de prise sur la question de Dieu, celle-ci ne dépend que de la décision de charité. Et ceci, de plusieurs manières. D'abord concernant l'existence, puisque la philosophie ne dispose pas (ou plus) d'un concept d'existence en général ; Kant l'a établi : l'existence n'offre aucune perfection réelle, qu'on pourrait décrire réellement et qui pourrait enrichir la définition d'une chose ou seulement la qualifier ; il ne s'agit que de sa position de fait, position qui se produit ou se constate, mais ne s'explique pas, ni ne dit rien de la chose. Cette inintelligibilité de l'existence en général éclate plus encore dans le cas où l'on appliquerait l'existence à Dieu ; car nous ne savons pas si l'ens (même le plus réel et le plus parfait que nous puissions concevoir) offre assez de dignité et donc de divinité, pour que nous soyons autorisés à l'attribuer à Dieu, comme nous l'attribuons à tout et n'importe quoi dans le monde. Que Dieu soit, cela n'en dit encore rien de divin, mais ne constitue qu'une aumône ontique, une pièce de petite monnaie, qu'on lui accordera sans peine, parce qu'elle ne nous coûte rien, dès lors que tout, y compris le presque rien, la reçoit toujours. Ensuite concernant l'essence : formellement, à supposer que Dieu se définisse, il se définira comme ce qui outrepasse toute définition id quo majus cogitari nequit ; il faut donc admettre qu'il échappe à tout concept d'essence. Thomas d'Aquin lui-même, citant Denys l'Aréopagite, rappelle que «[...] nous sommes 
joints à Dieu comme inconnu (quasi ignoto)», car «[...] nous connaissons ce qu'il n'est pas, mais ce qu'il est vraiment, cela nous demeure profondément inconnu (quid vero sit, penitus manet incognitum) $»^{31}$. De droit, celui qui croit en Dieu n'en sait pas plus sur l'essence de Dieu que celui qui n'y croit pas, et peut-être même moins, car il sait pourquoi il n'en sait rien - parce que « la vertu divine, comme elle est infinie, aucun entendement fini ne peut la comprendre, pas plus que son essence (non potest aliquis creatus intellectus comprehendere, sicut nec essentiam ejus) $»^{32}$. On peut certes viser Dieu comme l'acte pur d'être, puisque précisément il n'a pas d'autre essence que cet acte, mais en reconnaissant que nous ignorons entièrement ce que signifie en soi cet esse: "[...] hoc intelligitur de esse, quo Deus in seipso subsistit, quod nobis quale sit ignotum est, sicut ejus essentia $»^{33}$. Ou bien on dira, que le croyant en sait plus que l'incroyant, parce qu'il sait pourquoi il convient de ne connaître Dieu que comme inconnu - pour ne pas lui substituer une idole, à la mesure toujours finie de l'esprit qui s'imagine l'atteindre. Dès lors, si ni l'existence, ni l'essence ne conviennent à la connaissance de Dieu, de quoi se préoccupe la pensée théologienne ? De l'accès à la « charité », à laquelle seule, selon la Révélation du Christ, Dieu se reconnaît. À la charité, on n'accède que par la charité - on ne peut connaître Dieu qu'en l'aimant. Le vrai débat, le seul sérieux et rationnel, à propos de Dieu consiste à répondre à la question que le ressuscité adresse à Pierre : «M'aimes-tu?». Cette question relève d'un horizon infiniment étranger à la métaphysique, qui s'imagine que la demande «Dieu existe-t-il?» a la moindre pertinence, quand Dieu se révèle comme agapè. Si une philosophie de la religion entreprenait une telle herméneutique, elle permettrait à la pensée de remettre dans la lumière de l'agapè ce qui resterait, sinon, sous son propre soleil, ce soleil sous lequel chacun veut installer sa possession d'essence en toute injustice (Lévinas reprenant Pascal). Et cette opération interprétative peut se déployer en tous domaines, aussi bien sur la littérature que l'analyse politique ou économique.

Mais, dans tous les cas, la pensée, en l'occurrence chrétienne, traite son champ d'analyse comme, dans les services de renseignement, un officier traitant traite une " source » : il s'agit de lui faire dire ce qu'elle ne souhaitait d'abord pas dire, ce qu'elle ne savait parfois même pas savoir, d'en détourner son intention première pour faire apparaître ce qui aurait voulu rester cacher. Ainsi double-t-on un agent pour en faire un agent double. On pourrait aussi dire que la pensée chrétienne double ce que l'« esprit » dit, en le faisant parler la langue de la " charité ", alors qu'il ne croyait ne parler que la sienne - à la manière où l'on double un acteur dans un film, lui substituant une autre voix (voire un autre texte) dans la même langue, ou lui faisant dire la même chose dans une autre langue, détournée. Il se pourrait que la philosophie, voire la metaphysica ellemême puisse, malgré elle, nous enseigner beaucoup plus et bien autre chose que ce qu'elle pense savoir et veut expressément dire. Il s'agit de la laisser se contre-dire : non certes la faire se contredire en la réfutant ou la contestant; non certes non plus lui faire dire des contre-vérités ou prononcer des énoncés incohérents ; mais lui laisser dire autre chose, en marge, voire en opposition à ce qu'elle croit dire et devoir dire, la laisser parler en sens différent, divergent, voire contraire de ce qu'en première intention elle voulait dire.

La pensée chrétienne n'a nul besoin de la metaphysica, mais elle peut d'abord doubler le cap métaphysique du possible et de l'impossible, afin de redoubler les deux premiers ordres par l'ordre de la « charité » et ouvrir le champ propre de la théologie, en sorte que in fine la charité puisse doubler toute autre rationalité et soumettre tout à celui qui 
soumet tout au Père. Il s'agirait alors d'anagogie vers ce qui advient, plutôt que d'analogie avec ce qui est.

\section{NOTES}

1. Nous développons et corrigeons ici une première version de ce texte, parue in P. CapelleDumont, Jean Greisch, Jean-Luc Marion, Richard Kearney, Andreas Speer \& David Tracy (éd.), Métaphysique et christianisme, Paris, PUF, 2015 (reprise in W. Starnzynski \& A. Guimaraes Tadeu de Sores (éd.), « Dossiê Marion », Educacao e Filosofia, vol. 30, Minas Gerais, Uberlandia, 2016). Nous remercions notre ami P. Capelle-Dumont de nous l'avoir permis.

2. S. von Strochenau, Zugaben zur Philosophie der Religion, (1772), Augsburg, premier volume, suivi de plusieurs autres, d'après J. Greisch, qui précise que, «[...] nonobstant les prédécesseurs géniaux tels que Hume, Pascal et Spinoza, on peut difficilement parler de "philosophie de la religion" antérieurement au XVIII ${ }^{\text {e }}$ siècle, qui, rien qu'au point de vue lexical, se caractérise par une véritable profusion de publications traitant de la religion. " (Le buisson ardent et les lumières de la raison. L'invention de la philosophie de la religion, t. 1, Héritages et héritiers du XIX siècle, Paris, Cerf, 2002, p. 31). Voir L. Wallner, Der Verfasser der Religionsphilosophie Sigismund von Storchenau (1711-1797), Innsbruck, 1963 ; et, plus généralement, K. Feieres, Die Umprägung der natürlichen Theologie in Religionsphilosophie. Ein Beitrag zur deutschen Geistesgeschichte des 18. Jahrhunderts, Leipzig, Sankt Benno-Verlag, 1965 ; et J. Collins, The Emergence of Philosophy of Religion, NewHaven/London, Yale University Press, 1997.

3. «Auch bei dem Christentums soll man nicht fragen, wie habe ich zu denken, um es mit irgend einer Philosophie in Uebereinstimmung zu setzen, sondern umgekehrt, von welcher Art muß die Philosophie sein, um auch das Christentum in sich aufnehmen und begreifen zu können », F. W. J. Schelling, Philosophie der Offenbarung, Werke, t. 6, éd. M. Schröter, Munich, Beck, 1979, p. 34 ; trad. par J.-F. Marquet et J.-F. Courtine in Philosophie de la Révélation, XXV, t. 3, Paris, PUF, 1994, p. 55, modifiée.

4. F. Suarez, Opera omnia, Paris, L. Vivès, 1856, t. 25, p. 1.

5. D'où l'importance de la publication des Qucestiones super Metaphysicam, récemment commencée par O. Boulnois et D. Arbib, Jean Duns Scot. Questions sur la métaphysique, volume 1, Livres I à III, Paris, PUF, 2017.

6. O. Boulnois, Métaphysiques rebelles. Genèse et structures d'une science au Moyen-Age, Paris, PUF, 2013.

7. «Ea vulgo Metaphysica, sed aptius Ontologia sive scientia catholica, eine allgemeine Wissenschaft, et Philosophia universalis nominatur. [...]...denotat omne quod cogitari potest (distinctionis causa nonnullis vocatur Intelligibile) [...] nonnulla de Ente in prima et secunda acceptione [aliquid \& res/ substantia] praetermittemus, inchoaturi universalem philosophiam ab Ente cogitabili, quemadmodum a singulari incipiens prima philosophia nihil prius considerat Mente cogitante » (J. Clauberg, Metaphysica de Ente, Amsterdam, Kessinger, (1664), §§ 1, 2 \& 4, et Opera omnia Philosophica, Amsterdam (1691), Hildesheim, Olms, 1968, t. 1, p. 283. Voir M. Savini, Johannes Clauberg. Methodus cartesiana et ontologie, Paris, Vrin, 2011 et les travaux de J.-F. Courtine. De même Wolff : "Essentia definiri potest per id, quod primum de ente concipitur et in quo ratio continetur sufficiens » (Ontologia, Francfort/Leipzig, Renger, 1730, § 168). D'où suit logiquement la définition de la métaphysique par A. G. Baumgarten comme « [...] scientia primorum in humana 
cognitione principiorum» (Metaphysica, (1739), § 1, Stuttgart, éd. G. Gawlick \& L. Kreimendahl, Frommann-Holzboog, 2011, p.52), anticipant sur la transformation, en fait assez banale, de l'ontologie en connaissance par Kant. Et encore J. G. Fichte: «Das Wort Sein bedeutet unmittelbar immer schon ein Objekt des Denkens, ein Gedachtes Nun kommt ihm entweder auch eine Existenz, der Bestehen und Dauern, außer dem Denken zu, in der sinnlichen Wahrnehmung; dann ist ein reelles Sein bezeichnet, und man kann vom Gegenstande sagen: is ist. Oder es kommt ihm außer dem Denken kein anderes Sein zu; da ist die Bedeutung des Seins bloß die logische » (Rückerinnerungen, Antworten, Fragen, § 28, 1799, éd. F. Medicus, Fichtes Werke, Leipzig, Bd. III, p. 105 = J. G., Fichte, Sämtlichen Werke, 1845, Bd. V, p. 359).

8. I. Kant, Kritik der reinen Vernunft, A 247/ B 303.

9. E. Gilson, L'Etre et l'essence (1948), Paris, Vrin, 1962, p. 316.

10. Voir la déclaration solennelle et inaugurale de F. Nef: "Ces différentes mutations aboutissent à une définition très générale : l'ontologie est une discipline formelle qui traite des objets et du contenu des modèles qui nous permettent d'appréhender la réalité de la manière la plus générale et la la plus abstraite » (Traité d'ontologie pour les non-philosophes (et les philosophes), Paris, Gallimard, 2009, p. 20).

11. Selon la formule géniale de Kant, Lettre à Marcus Herz, après le 11 mai 1781, Correspondance, Paris, Gallimard, 1986, p. 181.

12. Ainsi T.-D. Humbrecht : «Le modèle interprétatif de la métaphysique est tout autre que celui de la modernité. [...]...lui [sc. saint Thomas] concéder une métaphysique d'un autre type, autre à beaucoup de points de vue, y compris dans son autonomie par rapport à l'architecture des sciences et au rôle de la théologie, mais cohérente [...]. Même restitué à son naturel, Thomas se présente comme l'un des cas possibles de modèles interprétatifs. Une métaphysique est possible, non moderne, précise quand même, d'une autre sorte de précision » (in P. Capelle-Dumont (éd.), Philosophie de Jean-Luc Marion. Phénoménologie, théologie, métaphysique, Paris, Hermann, 2014, p. 79 et nos réponses p. 131-133).

13. D. Hume, Enquiry concerning Human Understanding, $\mathrm{X}, 12$, Oxford/New-York, éd. T. L. Beauchamp, 1999, p. 173. Faut-il souligner que le refus du miracle ne dépend pas d'un rejet du christianisme? L'exemple de Malebranche (le plus éminent d'une grande troupe), qui récuse le miracle pour fonder en raison le christianisme, prouve le contraire.

14. «Quand donc aurons-nous enfin la séparation de la Métaphysique et de l'état; mais pour de bon, cette fois; la vraie, la bonne séparation; non pas toujours la séparation de la Métaphysique électoralement, politiquement la plus faible, en politique parlementaire, au profit et pour l'établissement gouvernemental de la Métaphysique électoralement, politiquement la plus forte, en politique électorale, mais définitivement la séparation de la métaphysique, forte ou faible, sans acception et sans exception, même électorale, même politique, et même parlementaire. [...] Nous avons le désétablissement des Églises. Quand aurons-nous le désétablissement de la métaphysique " (C. Péguy, De la situation faite au parti intellectuel, in Euvres en prose, Paris, éd. R. Burac, 1988, t. 2, p. 563 sq.). Certes directement liée à l'histoire polémique de la France au moment de la loi de séparation, cette remarque n'en mérite pas moins qu'on la prenne au sérieux dans la théorie.

15. «Le nettoyage philosophique de la religion catholique n'a jamais été fait. Pour le faire, il faudrait être dedans et dehors » (S. Weil, La pesanteur et la grâce, (1948), Paris, U.G.E, 1965, p. 134). Mais justement qui sait la frontière et la limite entre le dedans et le dehors?

16. "Quod impossibile est, existere nequit " et "Quod possibile est, illud existere potest " (C. Wolff, Ontologia, op. cit., §§ 132 \& 133). Voir : «Ens dicitur, quod existere potest, consequenter cui existentia non repugnat », § 134 , ibid.

17. «Nonnihil est aliquid. Repraesentabile, quidquid non involvit contradictionem, quidquid non est A et non-A, est possibile» (A.G. Baumgarten, Metaphysica, § 8, op. cit., p. 56). 
18. «Der höchste Begriff, von dem man eine Transzendentalphilosophie anzufangen pflegt, ist gemeiniglich die Einteilung in das Mögliche und das Unmögliche. Da aber alle Entteilung einen eingeteilten Begriff voraussetzt, so muß noch ein höherer angegeben werden, und dieser ist der Begriff von einem Gegenstande überhaupt » (I. Kant, Kritik der reinen Vernunft, A 290/B 346).

19. Luc 1,37 = Genèse 18, 27 (à propos de la naissance d'un enfant chez une femme stérile); Luc 18, 27 = Matthieu, 19, 26 (à propos de la dépendance du riche envers ses richesses) ; Marc 14, 36 (à propos de l'agonie à Gethsémani).

20. « Unde cum Deo nihil sit impossibile, oportet per ea quae in hoc mundo sunt impossibilia nos ad ipsum respicere, apud quem impossibilitas est necessitas - sicut infinitas in hoc mundo actu est impossibilis, sic magnitudo cujus non est finis, est necessitas illa, quae non ens seu nihil, ut sit, necessitat » (Nicolas de Cues, De possest, in L. Gabriel, Philosophisch-theologische Schriften, Wien, Herder, 1967, t. 2, p. 340).

21. "Quapropter tibi gratias ago, Deus meus, quia patefacis mihi, quod non est via alia ad te accendendi nisi illa, quae omnibus hominibus etiam doctissimis philosophis, videtur penitus inaccessibilis et impossibilis, quoniam tu mihi ostendisti te non posse alibi videri quam ubi impossibilitas occurrit et obviat " (Nicolas de Cues, De Visione Dei, c. IX, t. 3, in Jasper Hopkins, Nicholas of Cusa's Dialectical Mysticism: Text, Translation, and Interpretative Study of De Visione Dei, Minneapolis, The Arthur J. Banning Press, 1985, p. 132). Ce qui peut se transposer dans l'incompréhensibilité nécessaire de l'infini : « Apparuisti mihi Domine aliquando, ut invisibilis ab omni creatura, quia es Deus absconditus infinitus. Infinitas autem est incomprehensibilis omni modo comprehendendi » (Nicolas de Cues, c. XII, op. cit., p. 142); on retrouve alors la thèse de Descartes: «Idea infiniti, ut sit vera, nullo modo debet comprehendi, quoniam ipsa incomprehensibilitas in ratione formali infiniti continetur » (AT VII, 368). Sur tous ces points, voir une analyse plus détaillée dans J.-L. Marion, Certitudes Négatives, c. II, §§ 8-13, Paris, Grasset, 2010 , p. 87 sq.

22. Ueberlegungen XV (Cahiers noirs, 1939-1941, GA 96, p. 273), voir : « Si l'impossible - ce qui se soustrait à la computation - est devenu impossible, alors l'homme a falsifié sa plus petite petitesse en grandeur » (Ueberlegungen VI, GA 94, p. 496).

23. À Henri Cazalis, 29 mai 1867, Euvres complètes, éd. B. Marchal, Paris, Gallimard, 1998, "Pléiade", t. 1, p. 721. Déclaration surgie dans «le climat, noir, humide et glacial, de Besançon » (p. 714), où il entra «dans la Disparition suprême » (p. 715), mais où « la Poésie [lui] tient lieu de l'amour » (p. 715).

24. Pascal, Pensées, § 308, CEuvres Complètes, éd. L. Lafuma, Seuil, Paris, 1963, p. 540.

25. Pascal, De l'art de persuader, op. cit., p. 355 (nous soulignons). Il s'agit d'une citation implicite de saint Augustin : « Non intratur in veritatem, nisi per charitatem » (Contra Faustum, XXIII, 18, PL 42, 507), qui s'appuie sur Romains 5, 5, thèse illustrée, entre autres, par Grégoire le Grand (Moralia in Job, VI, 37, PL 75, 762 sq.), Pierre Lombard (In Sent. III, d. 24, c. 3), Guillaume de SaintThierry («[...] non tam ratio voluntatem, quam voluntas trahere videtur rationem ad fidem ", Speculum fidei, § 25, éd. M.-M. Davy, Paris, Vrin, 1959, p. 46), Gilbert de la Porrée (In Boethii de praedicationetrium personarum, PL 64, 1303), A. de Halès (Summa Theologiae, Introd., q. 2, 3, 3), etc. L'anti-thèse sur les " choses humaines " correspond à la définition du jugement vrai par Descartes (Meditatio IV, AT VII, p. 59). L'opposition est citée par Heidegger (Sein und Zeit, § 29, Tübingen, Max Niemeyer Verlag, 1976, p. 139) qui en détourne le sens ou ne veut pas l'admettre. Sur tout ceci, voir J.-L. Marion, D’ailleurs, le Révélation, Paris, Grasset, 2020, §§ 8-10.

26. Voir "La science toujours recherchée et toujours manquante ", in J.-M. Narbonne et L. Langlois, La Métaphysique. Son histoire, sa critique, ses enjeux, Paris/Québec, Vrin, 1999.

27. I. Kant, Kritik der reinen Vernunft, A 850.

28. Les revoir sous leur aspect véritable, donc aussi les récupérer, les ravoir, au sens familier de rendre à un ustensile sa propreté et sa propriété initiales à force de les nettoyer et de les polir.

29. Saint Augustin, Confessiones X, 23, 34, BA 14, Turnhout, Brepols Publisher, 1992, p. 202. 
30. Voir une esquisse («Dieu et l'ambivalence de l'être ", Conférence inaugurale de la "Chaire Dominique Dubarle », Transversalités, n. 125, Revue de l'Institut Catholique de Paris, janvier-mars 2013) et une lecture de la parabole du fils prodigue (en Dieu sans l'être, c. III, § 4, Paris, (1982), PUF, 2010, p.140 sq. ; et J.-L. Marion, Certitudes Négatives, c. IV, § 24, op. cit., p. 233 sq.) ou J.-L. Marion, D’ailleurs, le Révélation, § 19, op. cit.).

31. Saint Thomas d'Aquin, Contra Gentes III, c. 49.

32. Saint Thomas d'Aquin, Contra Gentes III, c. 56 (voir c. 55).

33. Saint Thomas d'Aquin, Contra Gentes II, c. 12. 